\title{
Kinematics of globular cluster systems in galaxy mergers
}

\author{
Christopher C. Hayward ${ }^{1}$, T. J. Cox ${ }^{1}$ and Lars Hernquist ${ }^{1}$ \\ ${ }^{1}$ Harvard-Smithsonian Center for Astrophysics, 60 Garden St., Cambridge, MA 02138, USA \\ email: chayward@cfa.harvard.edu,tcox@cfa.harvard.edu, lhernqui@cfa.harvard.edu
}

\begin{abstract}
We use numerical simulations to study how the spatial distribution and kinematics of globular cluster systems (GCS) are affected by galaxy mergers. Each progenitor galaxy is given a GCS modeled after the Galactic GCS. We then study how a major merger affects the spatial distribution and kinematics of the GCS. Here we present our methodology. Future work will investigate the effects of varying galaxy parameters, merger orbital parameters, and initial GCS number density profile and kinematics. We hope to be able to extract information about the merger history of a galaxy from observations of its GCS spatial distribution and kinematics.
\end{abstract}

Keywords. galaxies: formation, galaxies: evolution, galaxies: kinematics and dynamics, galaxies: star clusters, (Galaxy:) globular clusters: general, methods: n-body simulations

\section{Introduction}

Recent observations with multi-object spectrographs have enabled observers to obtain radial velocities for samples of hundreds of globular clusters (GCs) located in galaxies beyond the Local Group. (See Brodie \& Strader 2006 and Romanowsky 2006 for reviews.) The kinematics of elliptical galaxies GCS observed so far are very heterogeneous. Dissipationless simulations used to study the kinematics of GCS in mergers predict large amounts of rotation in the outer parts of the GCS (Hernquist \& Bolte 1993, Bekki, Beasley, Brodie, et al. 2005). Some GCS show no or very weak rotation out to large radii (e.g., NGC 1399, Richtler, Dirsch, Gebhardt, et al. 2004), but more work, both theoretical and observational, must be done before we can draw any definitive conclusions. We hope to extend previous theoretical work by using dissipational simulations to systematically explore the parameter space of GCS and merger properties and to study projection effects in order to understand the diverse GCS kinematics that have been observed.

\section{Methodology}

We perform simulations using the N-body/SPH code GADGET2 (Springel 2005). Our version includes radiative cooling of gas, star formation based on the Schmidt law (Schmidt 1959, Kennicutt 1998), the multiphase feedback model of Springel \& Hernquist (2003), and a central black hole (Springel, Di Matteo, \& Hernquist 2005). Each galaxy consists of a dark matter halo and a rotationally supported exponential disk with a fraction $f$ of the disk mass in gas and $1-f$ in stars. The dark matter halo is initialized to a Hernquist (1990) profile with effective concentration parameter 9 , spin parameter $\lambda$ $=0.033$, and circular velocity $V_{200}=160 \mathrm{~km} \mathrm{~s}^{-1}$. The progenitors are each composed of 120,000 dark matter particles and 80,000 disk particles. See Cox, Dutta, Di Matteo, et al. (2006) and references therein for a complete description of the simulations.

GCs in our simulations are unresolved, collisionless test particles. We randomly select 1000 dark matter particles from each progenitor's halo to have number density given by 
$n(r)=n_{0}\left(1+r / r_{C}\right)^{-\alpha}$ (a power law with a core term), following Djorgovski \& Meylan (1994), where $\mathrm{r}$ is the distance to the galaxy center. This profile provides the best fit to the Galactic GCs that are older than 10 Gyr and were probably not accreted by the MW, i.e., they are not on retrograde orbits or related to dwarf galaxies (Bica, Bonatto, Barbuy, et al. 2006). Our fiducial model uses $R_{C}=1.5 \mathrm{kpc}$ and $\alpha=4.0$. We set $n_{0}$ such that the total number of GCs between $r=0.1 \mathrm{kpc}$ and $r=200.0 \mathrm{kpc}$ is 1000 . Future work will study the effect of varying profile parameters and profiles, as the initial GCS density profile of a realistic progenitor spiral is not well-constrained.

We make our initial GC selection without regard to kinematics. A GCS selected in this manner is supported primarily by velocity dispersion (typically $V / \sigma<0.05$ ). Future work will study rotationally-supported GCS.

\section{Future Work}

We plan to study how the initial GCS profile and kinematics, merger orbital parameters, gas fraction, and projection affect the resulting spatial distribution and kinematics of the GCS of the remnant. We will compare the GCS kinematics to the kinematics of the stars from the progenitors and stars formed in the merger and study how well GCs trace the dark matter distribution. Future work will also include prescriptions for GC formation and destruction and study the effect of these processes on the GC luminosity function.

\section{Acknowledgements}

This material is based upon work supported under a National Science Foundation Graduate Research Fellowship (CCH).

\section{References}

Bekki, K., Beasley, M. A., Brodie, J. P., \& Forbes, D. A. 2005, MNRAS 363, 1211

Bica, E., Bonatto, C., Barbuy, B., \& Ortolani, S. 2006, A\&A 450, 105

Brodie, J. \& Strader, J. 2006, ARAA 44, 193

Cox, T. J., Dutta, S. N., Di Matteo, T., Hernquist, L., Hopkins, P. F., Robertson, B., \& Springel, V. 2006, ApJ 650, 791

Djorgovski, S. \& Meylan, G. 1994, AJ 108, 1292

Hernquist, L. 1990, ApJ 356, 359

Hernquist, L. \& Bolte, M. 1993, in: G. H. Smith \& J. P. Brodie (eds.), The globular clustersgalaxy connection, Proc. of the 11th Santa Cruz Summer Workshop in Astronomy and Astrophysics (San Francisco: ASP), vol. 48, p. 788

Kennicutt, Jr., R. C. 1998, ApJ 498, 541

Richtler, T., Dirsch, B., Gebhardt, K., Geisler, D., Hilker, M., Alonso, M. V., Forte, J. C., Grebel, E. K., Infante, L., Larsen, S., Minniti, D., \& Rejkuba, M. 2004, AJ 127, 2094

Romanowsky, A. J. 2006, in: S. S. Larsen \& T. Richtler (eds.), Globular Clusters - Guides to Galaxies, ESO/Springer Conference Proceedings, in press, astro-ph/0609251

Schmidt, M. 1959, ApJ 129, 243

Springel, V. 2005, MNRAS 364, 1105

Springel, V., Di Matteo, T., \& Hernquist, L. 2005, MNRAS 361, 776

Springel, V. \& Hernquist, L. 2003, MNRAS 339, 289 